\title{
Gram-Positive and Gram-Negative Subcellular Localization Using Rotation Forest and Physicochemical-Based Features
}

\author{
Abdollah Dehzangi ${ }^{1,2}$, Rhys Heffernan ${ }^{1}$, James Lyons ${ }^{1}$, Alok Sharma ${ }^{1,3}$, \\ Kuldip Paliwal ${ }^{1}$, and Abdul Sattar ${ }^{1,2}$ \\ 1 Institute for Integrated and Intelligent Systems (IIIS), \\ Griffith University, Brisbane, Australia \\ 2 National ICT Australia (NICTA), Brisbane, Australia \\ 3 School of Engineering and Physics, University of South Pacific, Fiji \\ \{a.dehzangi, r.heffernan, j.lyons, a.sharma, \\ k.paliwal,a.sattar\}@griffith.edu.au
}

\begin{abstract}
The functioning of a protein relies on its location in the cell. Therefore, predicting protein subcellular localization is an important step towards protein function prediction. Bacterial proteins are considered among the most important proteins that play a wide range of useful and harmful roles. They generally can be divided into two groups namely: Gram-positive and Gram-negative. In this study, we aim at solving Gram-positive and Gram-negative bacterial proteins subcellular localization. Recent studies have shown that using Gene Ontology (GO) for feature extraction can improve the prediction performance. However, for newly sequenced proteins, the GO is not available. Therefore, for these cases, the prediction performance of GO based methods degrade significantly. Besides, the impact of other sources of features such as physicochemical, and evolutionary information to extract local information to tackle this problem, have not been explored adequately.

In this study, we develop a method to effectively employ physicochemical and evolutionary-based information in the protein sequence. To do this, we propose two new segmentation-based feature extraction methods, namely: overlapped segmented density and overlapped segmented autocorrelation. We extract these feature groups by dividing the protein sequence into several segments and extract density and autocorrelation information for each segment in the cumulative, overlapping manner. These feature groups are extracted to capture potential discriminatory information based on physicochemical properties of the amino acids to tackle Gram-positive and Gram-negative subcellular localizations. To incorporate evolutionary information as well, we extract overlapped segmented density and overlapped segmented autocorrelation feature groups from the transformed protein sequence using Position Specific Scoring matrix (PSSM).

We also extract two evolutionary based feature groups, namely: semi composition and auto covariance feature groups. These features groups are extracted to add more evolutionary information to our extracted features. We investigate the effectiveness of our proposed feature extraction
\end{abstract}


techniques using 10 attributes. These attributes have been experimentally selected among a wide range of (117 attributes) physicochemical attributes. We also employ Rotation Forest classification technique for our task. Rotation Forest is a newly proposed classifier to build ensemble classifier. It is based on the bagging technique and aims at increasing the diversity in the ensemble classifier. Despite its promising performance for similar studies, it has not been used for protein subcellular localization prediction problem (Figure 1). By applying Rotation Forest to our extracted features, we enhance Gram-positive and Gram-negative subcellular localization accuracies up to $3.4 \%$ better than previous studies which also used Gene Ontology for feature extraction.

Keywords: Protein Subcellular Localization, Gram-positive, Gramnegative, Feature Extraction, Physicochemical-based Features, Rotation Forest.

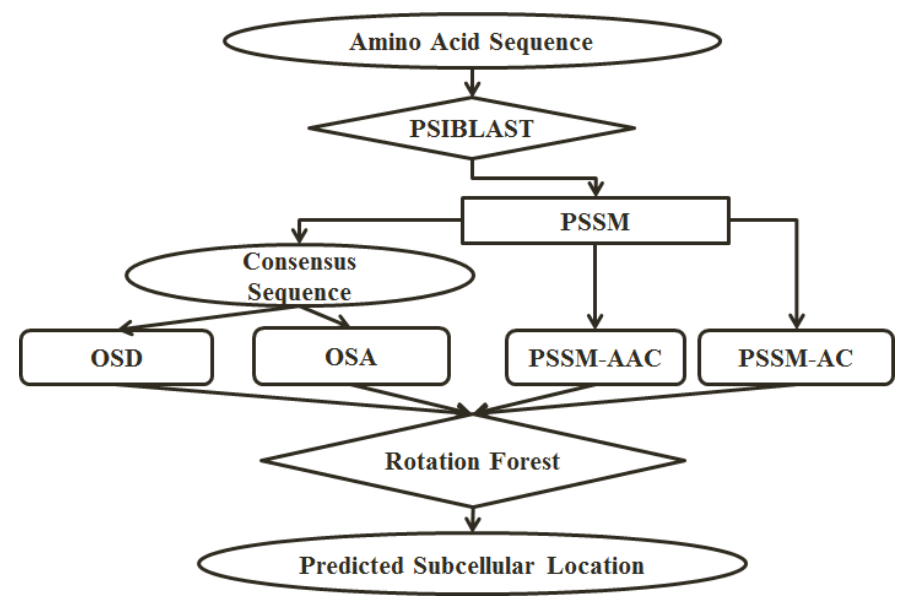

Fig. 1. The overall architecture of our proposed approach 